\title{
NEOGÉNESIS: \\ UN MODO DE ABORDAJE \\ DE LO TRAUMÁTICO
}

Magdalena Lema

Licenciada en Psicología de la UCU

Especialista en Psicoterapia Psicoanalítica del IUPA

Miembro de la AUDEPP

Correo electrónico: magdalena.lema@gmail.com

ORCID: 0000-0001-6731-6470

Silvia Tejeria

Licenciada en Psicología de la UDELAR

Miembro Habilitante de la AUDEPP

Correo electrónico: silviatejeria@gmail.com

ORCID: 0000-0002-3268-7789 


\section{Resumen}

En este artículo trabajamos el concepto de neogénesis, acuñado por Silvia Bleichmar. Partimos de las conceptualizaciones de esta autora acerca de los efectos traumáticos de lo real sobre el psiquismo, y articulamos los conceptos de trauma y violencia. Estos conceptos suponen una fuerte revisión teórica de ciertos postulados epistemológicos con su correlato de reformulaciones en la práctica clínica. Si bien la violencia no es un concepto específicamente psicoanalítico, en la clínica somos testigos de su incidencia en el sufrimiento humano y de su acción devastadora en el funcionamiento habitual de los sujetos. Bleichmar se ocupa del impacto singular y subjetivo del hecho traumático, y se pregunta cómo es afectado el yo en sus funciones y cuáles son las modalidades de respuesta del sujeto ante lo traumático. Cuando las respuestas del sujeto ante el embate de lo traumático escapan a las posibilidades de encadenamientos simbólicos y la represión no organiza, es necesario instrumentar una clínica especial que propicie la escucha de aquellos indicios de la experiencia real, restos metonímicos que hacen signo para el analista. En este trabajo de neogénesis, la intervención terapéutica no se reduce a encontrar lo que ya estaba, sino que intenta producir nuevas condiciones de simbolización.

Palabras clave: neogénesis, trauma, violencia, realidad

\section{NEOGENESIS: AN APPROACH TO TRAUMA}

\section{Abstract}

In this article, we work on the concept of Neogenesis, coined by Silvia Bleichmar. We start by considering Bleichmar's conceptualizations about the traumatic effects of the real on the psychism, by articulating the concepts of trauma and violence. These concepts imply a strong theoretical revision of certain epistemological postulates with their correlation of reformulations in clinical practice. Although violence is not specifically a psychoanalytic concept, in our practice we are witnesses to its incidence in human suffering and its devastating action in the habitual functioning of the subjects. Bleichmar deals with the singular and subjective impact of the traumatic event, questioning how the self is affected in its functions, and what are the modalities of the subject's response to the traumatic. When the responses of the subject to the onslaught of the traumatic escape the possibilities of symbolic chaining, and repression does not organize, it is necessary to implement a special clinic that encourages the listening of those signs of real experience, metonymic remains that become a sign for the analyst. In this work of Neogenesis, therapeutic intervention is not reduced to finding what was already there, but attempts to produce new symbolization conditions.

Keywords: neogenesis, trauma, violence, reality 


\title{
INTRODUCCIÓN
}

\author{
Yo muero extrañamente... No me mata la Vida, \\ no me mata la Muerte, no me mata el Amor; \\ muero de un pensamiento mudo como una herida.
}

Delmira Agustini (1965).

El abordaje de las situaciones traumáticas plantea desafíos clínicos particulares que implican la necesidad de poner a trabajar nuestras teorías para generar nuevas posibilidades de intervención. Consideramos de gran relevancia el concepto de neogénesis, acuñado por Silvia Bleichmar (1993), en la medida en que se trata de un modo de considerar los efectos psíquicos de lo traumático, que abre, destraba y ofrece posibilidades creativas y simbolizantes. La riqueza de este concepto nos permite abordar especialmente la articulación de las nociones de violencia y trauma, que serán desarrolladas a lo largo de este trabajo.

El concepto de trauma se encuentra en el origen mismo del psicoanálisis, casi como piedra angular de todo el edificio conceptual, aunque no siempre utilizado con un sentido unívoco. Violencia es un concepto ajeno al psicoanálisis, pero que entendemos se encuentra en algún tipo de articulación con nuestra tarea, en tanto somos testigos de la fuerza destructiva de sus efectos.

Violencia y trauma. Entonces, ¿cómo articularlos? En la vieja idea freudiana de un «origen traumático de la neurosis», el concepto de aprèscoup marca la complejidad de esa articulación (Freud, 1985). Lo que nos interesa destacar es que la forma en que pensamos en psicoanálisis la 
relación entre los fenómenos psíquicos y lo real no es un asunto menor, sino un verdadero problema epistemológico.

En esta línea, Bleichmar ha pensado con rigor psicoanalítico acerca de los efectos traumáticos de lo real sobre el psiquismo. Es decir, los diversos modos en que el embate de lo acontencial actúa sobre el sujeto y genera sufrimiento, descompone y desarticula su funcionamiento habitual. Desde una fuerte revisión teórica de ciertos postulados psicoanalíticos, entiende los tiempos míticos de la represión primaria como tiempos histórico-vivenciales reales. Por lo tanto, es posible identificar los momentos fundantes de estructura psíquica y por ello es posible desarrollar nuevas herramientas de intervención.

Desde Nuevos fundamentos para el psicoanálisis (1987) y La prioridad del otro (1996), Jean Laplanche establece un giro fundamental en la epistemología psicoanalítica: el otro como punto de partida para comprender la constitución psíquica. La situación antropológica fundamental dada por la necesidad de la cría humana —en su desauxilio inicial— de un adulto que se ocupe del cuidado de la vida y tome a su cargo los procesos de humanización marca el carácter estructurante de la relación con el otro real. A partir de aquí, el problema de la constitución psíquica está dado más bien por cómo el psiquismo se cierra —es decir, cómo consigue una delimitación y una diferenciación-, que por cómo se abre al mundo. La realidad no es secundaria, sino primordial. Por ello, la tópica psíquica se construye en el marco de la tópica intersubjetiva que el Edipo define con su estructura. El ser humano llega a un mundo ya pautado por ciertas legalidades que van a determinar el modo como el adulto se emplaza frente al niño, desde una doble función: la inscripción de la sexualidad y, al mismo tiempo, la pautación de los límites de su apropiación del cuerpo del infans como lugar de goce.

En este contexto, habría que diferenciar una teoría traumática de la constitución psíquica, de aquellos traumatismos específicos con efecto 
desestructurante, que someten al psiquismo a un monto de excitación inderivable, a experiencias no metabolizables, a la imposibilidad de ligadura, y que constituyen verdaderos procesos de desmantelamiento psíquico. $Y$ diferenciar también los microtraumas de la vida cotidiana, que fuerzan procesos más o menos exitosos y creativos de elaboración, de aquellas otras situaciones de real violentamiento. Situaciones ante las cuales el sujeto no puede responder con el equipamiento defensivo habitual, situaciones que implican un desmantelamiento defensivo, una efracción de la membrana psíquica o una ruptura de la continuidad existencial, al decir de Donald Woods Winnicott.

En La desconstrucción del acontecimiento (2006), Bleichmar cita la comunicación preliminar de Freud, que plantea: «En calidad de tal [trauma psíquico], obrará toda vivencia que suscite los afectos penosos del horror, la angustia, la vergüenza, el dolor psíquico y, desde luego, la sensibilidad de la persona afectada. Y agrega que lo vivido, o su recuerdo, opera a modo de cuerpo extraño que aún mucho tiempo después de su intrusión conserva eficacia presente» (Freud, 1895, en Bleichmar, 2006, s.p.). *

¿Cómo es afectado el yo por «la incidencia atacante de lo real»? Según Bleichmar (2014), «el impacto de lo traumático pone en riesgo, en mayor o menor medida, dos grandes aspectos de la organización del yo y de su función: la autopreservación y la autoconservación» (p.40); es decir, la forma como el yo se representa la conservación de la vida y sus riesgos, por un lado, y, por otro, la forma como se siente en riesgo respecto de los enunciados identificatorios que lo constituyen. Normalmente, estas dos dimensiones van juntas, pero los traumatismos marcan su diferenciación. En términos generales, en las catástrofes naturales está en riesgo la autoconservación y en las a catástrofes históricas se ven constantemente

* El destacado es nuestro. 
afectados los enunciados identitarios constitutivos del yo, lo cual pone en riesgo la autoestima y lleva a procesos de desidentificación.

Se trata de poner de relieve la capacidad de lo acontencial (en tanto real histórico) de producir efectos, en la medida en que resulta imposible de engarzar en los sistemas psíquicos previos, ya que colocan al yo en riesgo de sobrevivencia biológica o simbólica.

Frente a estas experiencias, el psiquismo puede responder bajo dos modalidades, que no son excluyentes: al modo de lo originario y al modo de lo arcaico.

La cadena de respuestas al modo de lo originario implica todo el repertorio de formaciones sintomáticas sobre las que es posible seguir una traza simbólica, en tanto que la posibilidad de ligar se mantuvo y, aun dentro de la patología, el sujeto puede encontrar algún tipo de equilibrio. Podemos decir que la represión opera y que existe una malla simbólica que hace posible tramitar lo vivido.

Cuando, en cambio, la respuesta es al modo de lo arcaico, nos encontramos con otro fenómeno, en el que la represión no organiza y, más bien, las representaciones avanzan, desconectadas o desligadas, sobre un yo que se siente inerme para manejarlas. Se trata, en este caso, de los restos de lo real vivido, pero al modo de un material en bruto, desengarzado, que requiere de algo totalmente diverso de la interpretación simbólica. Supone ingresar en una clínica especial, que dé lugar a la escucha de aquellos indicios de la experiencia real, en la búsqueda de simbolizaciones de transición que aporten algún nivel incipiente de ligadura.

Entonces, es necesario preguntarnos: ¿de qué modo se produjo el desarme de la malla psíquica?, ¿qué opera de la represión originaria?, ¿qué restos de posibilidades de simbolización existen?, ¿qué de lo real vivido insiste, desarticulando y rompiendo? 


\section{NEOGÉNESIS}

El concepto de neogénesis viene a ampliar el repertorio de respuestas posibles ante la insuficiencia de la interpretación simbólica para cercar lo arcaico. Se trata de un movimiento que pretende generar las condiciones básicas para la simbolización posterior, en lugar de dar significados a priori.

Normalmente, el análisis trabaja sobre lo secundariamente reprimido, sobre aquellos contenidos que alguna vez fueron palabra y que, por lo tanto, son recuperables por medio de la asociación libre. Opera sobre el levantamiento de represiones y el develamiento del sentido oculto, ya que, en efecto, lo secundariamente reprimido es lo único sobre lo que el sujeto puede recomponer las cadenas asociativas, en la medida en que alguna vez esos contenidos formaron parte de la trama del lenguaje articulado.

Pero ¿qué ocurre cuando la organización defensiva del sujeto estalla espontáneamente por efecto de situaciones imposibles de tramitar o metabolizar con el equipamiento defensivo disponible?

No es posible seguir los mismos procedimientos cando se trata de lo que nunca cayó bajo el efecto de la represión. Es este caso, se trata de cercar esas representaciones, «de recomponerlas cuando avanzan y, si no avanzan, dejarlas dormidas» (Bleichmar, 1999, p. 105). Se aplica plenamente en este punto lo que plantea José Luis Martí Quirós (2004), para quien la escucha analítica es la «escucha de un silencio», es la escucha de lo que no puede ser dicho.

En ocasiones, un sonido, un olor, un dibujo de un niño, un dato perceptivo, aislado de cualquier ensamblaje (un signo de percepción, de acuerdo a los términos freudianos), puede ser capaz de producir perturbaciones, de desencadenar repeticiones o compulsiones, sin un aparente sentido. El signo de percepción queda anudado a la experiencia traumática como 
resto metonímico. No es inconsciente, o sea que no ha caído bajo los efectos de la represión, y constituye el indicio privilegiado que lleva al trauma. El resto indiciario de lo real vivido no es un recuerdo, ya que para que exista recuerdo tiene que haber un sujeto que narre la historia, y estos son elementos que se encuentran fuera de la historización. Más que recuerdos, podría decirse que se trata de reminiscencias de algo que permanece como resto inmodificado que determina el estancamiento de la producción psíquica.

\section{PENSANDO DESDE LA CLINICA}

La consulta de una mujer de 38 años nos ayuda a ejemplificar este tipo de intervenciones. Es una profesional universitaria que ha pasado por una penosa situación de violencia ejercida por su expareja, lo que deriva en reiteradas denuncias, en la judicialización del caso y en la prescripción de medidas severas: restricción de acercamiento y consiguiente colocación de tobillera electrónica al agresor. Asimismo, la pericia forense realizada a este hombre explicita la presencia de un trastorno antisocial de la personalidad, con fuertes componentes de manipulación, impulsividad, ausencia de sentimientos de culpabilidad, imposibilidad de empatía...; en fin, con la posibilidad de incurrir en acciones violentas que ponen en riesgo la integridad física del otro.

Esta mujer tiene con este hombre una hija de 6 años de edad. Acudió a la consulta dos meses después de haber reanudado, por su cuenta, el contacto con el padre de la niña, quien en el momento actual concurre a su casa a visitarla, lo cual ha generado nuevamente situaciones de violencia. A su vez, el padre ha llevado a su hija a dormir a su casa sin acuerdo de la madre. La situación se torna inmanejable para esta mujer, y consulta. 
En algún momento del proceso terapéutico llegamos a un punto en el que pregunto: «¿Qué pasó? ¿Por qué habrá sido que lo llamaste de nuevo?».

La paciente rápidamente responde: «Es que, pobrecita Nati, yo la veía tan solita...».

El indicio «tan solita» da lugar a una intervención bajo la forma de pregunta: «Y tú, ¿te has sentido así alguna vez?». Esta intervención, que opera al modo de simbolización de transición, me lleva a una escena desconocida hasta el momento. Conduce al despliegue de la situación traumática histórica de la paciente: el maltrato infantil, el padre que no tuvo, un abuelo protector que murió cuando ella tenía 5 años, el abandono de la madre, la vida con una tía maltratadora en medio de carencias muy severas.

Hay que decir que el indicio sería aquello que hace signo para alguien, en este caso para el analista que escucha. No es un signo para el paciente.

Entonces, el modo de engarzar estos indicios a la trama psíquica y reducir sus efectos destructivos es a través de la elaboración de simbolizaciones de transición que, como verdaderos autotrasplantes psíquicos, hagan posible recomponer y articular lo desgarrado.

Neogénesis, tal como sostiene Bleichmar (1999), sería entonces aquel «movimiento que en la práctica no se limita a recuperar lo ya existente, sino que intenta generar nuevas condiciones de simbolización, abrir nuevas posibilidades de vida» (p.12). Y la autora agrega: «la intervención del analista no se reduce a encontrar lo que ya estaba, sino a producir elementos nuevos de recomposición y de articulación que den un producto diferente del preexistente» (p.37).

En estos casos, la interpretación simbólica obtura la posibilidad de establecer un nexo más profundo con lo vivenciado, que es absolutamente singular. Las simbolizaciones de transición hacen posible el nexo, permiten la apropiación de un fragmento representacional que no puede ser 
aprehendido mediante la libre asociación, cuya significación escapa, pero insiste de manera compulsiva.

A diferencia de la construcción, no ofrece un sentido más o menos cerrado proporcionado desde la función sintética del analista, sino que más bien implanta contextos que han sido relatados y que son conocidos en el interior del proceso analítico, pero que no han sido relacionados con el elemento emergente. Se trata de restituir el fragmento indiciario a la escena de la que formó parte, para dar lugar al paso de la metonimia a la metáfora.

\section{LA FUNCIÓN DEL TERAPEUTA}

Teresa Sánchez (2017) nos recuerda que Julia Kristeva decía: «Un analista que no descubre en cada uno de sus pacientes una nueva enfermedad del alma, no lo escucha en su verdadera singularidad» (p.2).

En el terreno de lo traumático, la búsqueda de lo singular es, para el analista, la disposición a ingresar en el terreno de lo irrepresentable o de lo impensable, en la medida que no estamos en el terreno de lo secundariamente reprimido y, por ello, recuperable desde su encadenamiento simbólico.

La idea de figurabilidad psíquica, que desarrollan con gran profundidad César y Sara Botella (2001), nos ayuda a pensar «en esa zona psíquica-prepsíquica situada a uno y otro lado del mundo de las representaciones, especialmente el de las representaciones de la palabra, de las formaciones preconscientes, de la racionalidad» o bien en la «memoria sin recuerdo» o en lo que los autores llaman tras-país. Este sería el de los «acontecimientos que no han podido adquirir la condición de representación», ya sea que se refieren a un pasado, como a un presente que «escapa a la posibilidad de ser puesto en historia-relato debido al fracaso del Trabajo de Representancia» (Botella, 2014, pp.1-3). 
Acceder a este universo prepsíquico requiere de una escucha fina e implicada, que nos coloca en un terreno complejo y que en ocasiones nos lleva al límite de nuestra capacidad de procesamiento y a nuestros propios impensados. Al decir de Botella y Botella (2001), se trata de una escucha particular, que implica en la mente del analista procesos regredientes - al modo del trabajo del sueño— que hacen posible un trabajo de figurabilidad psíquica.

Para finalizar, acordamos con lo que plantea Susana García Vázquez (2005) con respecto a que nuestra tarea «requiere libertad, modestia y rigurosidad. Libertad para poder pensar y apelar a distintos recursos, modestia para no olvidar nuestros límites, los límites de nuestro instrumento y los del paciente, y rigurosidad en la reflexión que la clínica diariamente obliga» (s. p.).

\section{REFERENCIAS BIBLIOGRÁFICAS}

Agustini, D. (1965). Antología. Clásicos uruguayos (vol. 69). Montevideo: Biblioteca Artigas.

Bleichmar, S. (1993). La fundación de lo inconsciente. Destinos de pulsión, destinos del sujeto. Buenos Aires: Amorrortu.

Bleichmar, S. (1999). Clínica psicoanalitica y neogénesis. Buenos Aires: Amorrortu.

Bleichmar, S. (2006). La desconstrucción del acontecimiento. En L. Glocer Fiorini (comp.), Tiempo, Historia y Estructura. Su impacto en el psicoanálisis contemporáneo. Buenos Aires: Lugar y APA. 
Bleichmar, S. (2014). Las teorías sexuales en psicoanálisis. Qué permanece de ellas en la práctica actual. Buenos Aires: Paidós.

Botella, S. y Botella, C. (2001). La figurabilidad psíquica. Buenos Aires: Amorrortu.

Botella, C. (2014). Sobre el trabajo de figurabilidad. Asociación Internacional Para el Estudio y Desarrollo de la Mentalización.

https://revistamentalizacion.com/ultimonumero/botella.pdf

FREUd, S. (1985). Obras completas. Estudios sobre la histeria (J. Breuer y S. Freud) (1893-1895) (vol. II). Buenos Aires: Amorrortu.

García Vázquez, S. (2005). Trauma psíquico y método psicoanalítico. Revista Uruguaya de Psicoanálisis, 100, 149-169.

https://www.apuruguay.org/revista_pdf/rup100/100-garcia.pdf

LAPLANCHE, J. (1987). Nuevos fundamentos para el psicoanálisis. La seducción originaria. Buenos Aires: Amorrortu.

Laplanche, J. (1996). La prioridad del otro en psicoanálisis. Buenos Aires: Amorrortu.

Martí Quirós, J. L. (2004). La escucha alienada. Revista de Psicoanálisis de la Asociación Psicoanalitica de Madrid, 43, 157-162.

SÁnchez, T. (2017). Cambios en la escucha psicoanalítica de los trastornos ligados a la hipermodernidad. Aperturas psicoanalíticas, 56. http://shorturl.at/sFMQ3 